\title{
Sistema de proyección de la demanda. Caso práctico de predicción automatizada en empresas de venta por catálogo
}

\section{ANTONIO JOSÉ BOADA ${ }^{a}$}

\begin{abstract}
RESUMEN El presente artículo expone los resultados de un trabajo académico realizado en una empresa manufacturera de consumo masivo en el área cosmética, bajo el estilo de venta por catálogo o venta directa. A partir de este, se logró diseñar una herramienta automatizada para predecir productos por catálogo, en función de diversas variables de mercadotecnia. Dicho estudio se sustentó en un análisis detallado de las variables causales, impulsadoras e inhibidoras de la demanda, desarrollando posteriormente un software denominado sistema de proyección de la demanda, herramienta creada con la finalidad de automatizar los procesos inherentes a las estimaciones-predicciones de ventas, procesos fundamentados estadística y matemáticamente mediante variables sólidas en referencia al producto, precio, fabricación y facturación, y variables blandas correspondientes a planificaciones futuras de gerencias estratégicas como mercadeo y ventas.

La dinámica de las empresas de venta por catálogo impulsa a generar amplias estrategias de planificación futura, con tiempos cortos de reacción, que originan la adquisición de inventarios para periodos únicos, derivando así en estrategias de predicción más afinadas y automatizadas.
\end{abstract}

PALABRAS CLAVE predicciones de ventas, regresión, sistema de proyección, técnicas bayesianas.

\section{HISTORIA DEL ARTÍCULO}

¿CÓMO CITAR?:

Boada, A.J. (2017). Sistema de proyección de la demanda. Caso práctico de predicción automatizada en empresas de venta por catálogo. Perspectiva Empresarial, 4(1), 23-41. http://dx.doi.org/10.16967/rpe. v4n1a2

RECIBIDO: 9 de agosto de 2016 APROBADO: 9 de diciembre de 2016

CORRESPONDENCIA:

Antonio José Boada. Calle 77 Sur No. 40 - 165. Piso 6. Área de Ciencias Básicas Tel. (4) 3056100 C.P. 055450 Sabaneta-Antioquia, Colombia.

a Docente en CEIPA Business School y en la Universidad Simón Bolívar, Colombia - Venezuela. Correo electrónico: antonio_boada@virtual.ceipa.edu.co, antonio.boada@ceipa.edu.co 
¿CóMO CITO EL ARTÍ́CULO? HOW TO CITE THIS PAPER?

\section{CHICAGO:}

Boada, Antonio José. 2017. "Sistema de proyección de la demanda. Caso práctico de predicción automatizada en empresas de venta por catálogo". Perspectiva Empresarial 4(1): 23-41. http:// dx.doi.org/10.16967/rpe. v4n1a2

MLA:

Boada, Antonio José. "Sistema de proyección de la demanda. Caso práctico de predicción automatizada en empresas de venta por catálogo". Perspectiva Empresarial 4.1 (2017): 23-41. Digital. http:// dx.doi.org/10.16967/rpe. v4n1a2

\section{Demand Forecast Systems. Automated Forecast Practical Case in Catalog Sales Companies}

ABSTRACT This paper presents the results of an academic research conducted on a mass consumption, cosmetics manufacturing company that reaches its clients through catalogs or direct sales. Based on the research, it was possible to design an automated tool that predicts the products to be placed in a catalog based on several marketing variables. The research was based on a detailed analysis of the causal, fostering and inhibiting variables of the demand and, then a software called demand forecast system was developed to automatize the process to statistically and mathematically estimate/forecast sales using solid variables in terms of product, price, manufacturing, and invoicing, as well as soft variables for future planning of strategic managerial skills such as marketing and sales.

The dynamics of a catalog sales company is ideal to generate several future planning strategies, with short reaction terms that can originate the acquisition of inventories for oneoff seasons, which results in more accurate and automated forecast strategies.

KEYWORDS sales forecast, regression, forecast system, Bayesian techniques

\section{Sistema de projeção da demanda. Caso prático de predição automatizada em empresas de venda de catálogo}

RESUMO Este artigo apresenta os resultados de um trabalho acadêmico realizado em uma empresa manufatureira do setor de consumo massivo na indústria cosmética, sob o estilo de revenda de catálogo ou venda direta. Daqui estruturou-se uma ferramenta automatizada para visualizar produtos de catálogo em função de diferentes variáveis de marketing. Esse estudo se sustentou em uma análise detalhada das variáveis causais, impulsadoras e inibidoras da demanda, desenvolvendo depois um software chamado sistema de projeção da demanda, criado para automatizar os processos próprios das estimativas-predições de vendas, processos fundamentados estatística e matematicamente por meio de variáveis sólidas no que respeita ao produto, preço e fabricação e variáveis suaves no que respeita aos planejamentos futuros de gerências estratégicas como marketing e vendas.

A dinâmica das empresas de revenda de catálogo impulsa a gerar estratégias amplas de planejamento futuro, com tempos curtos de reação, que originam a aquisição de estoques para periodos únicos, acabando em estratégias de predição mais adequadas e automatizadas.

PALAVRAS CHAVE Predições de vendas, regressão, sistema de projeção, técnicas bayesianas. 


\section{Introducción}

Los pronósticos son vitales para toda organización mercantil y para toda decisión administrativa importante. Presentan la base de los planes a largo plazo de la empresa. En las áreas funcionales de finanzas y contabilidad, los pronósticos son el cimiento para la planeación del presupuesto y el control de los costos. El departamento de Marketing depende de los pronósticos de ventas para cuantificar sus planes de productos nuevos y regulares, las estrategias de ventas y la valoración de los impactos promocionales que optimicen la toma de decisiones fundamentales. El personal de producción y el de operaciones usan los pronósticos para tomar decisiones de manera periódica, respecto a la selección de procesos, la planeación de la capacidad y la disposición física de las instalaciones, así como para las decisiones rutinarias sobre los planes de producción, los programas y los inventarios (Chase, Jacobs \& Aquilano, 2005).

Actualmente se utilizan diversas técnicas de análisis estadístico multivariante en el ámbito de mercadeo e investigación de mercados, tales como estudios estratégicos de segmentación de mercados, análisis de correlación, análisis discriminante, escalas multidimensionales, análisis de clúster, entre otros (Prieto, 2013); sin embargo, el trabajo de Prieto se focaliza en el diseño estructural de un modelo estadístico de predicción, que pudiera derivarse en la consolidación de una herramienta automatizada en Visual Basic con base de datos Access, que permitiera a la empresa ejecutar, en tiempo real, proyecciones de demanda futura, alimentadas por variables causales de mercadotécnicas previamente valoradas según la información histórica particular de la compañía.

En el caso particular de las empresas de venta por catálogo, el comportamiento de la demanda presenta diferencias con el estilo tradicional retail, ya que en el primer tipo de empresas la compañía posee el control de las formas de exponer y promocionar los productos dentro de su catálogo de ventas, el cual tiene un tiempo de vigencia determinado. De allí que la búsqueda de una aplicación automatizada para poder predecir la demanda futura de unidades en empresas con estilo de ventas por catálogo, siempre ha sido una necesidad para poder administrar la demanda futura con eficiencia en las áreas de planificación, logística, compras y producción.

Este proyecto estadístico académico-industrial se generó así, con la finalidad de analizar estadísticamente la función de demanda en los productos de una empresa manufacturera de consumo masivo, con estilo de venta directa (Avon Cosmetics), infiriendo así el comportamiento de los artículos en el tiempo (durante la vigencia de los catálogos de ventas), en su segmentación más detallada a nivel de SKU (Stock Keeping Unit (SKU). (Boada, 2013).

Desarrollando un modelo matemático predictivo, y fundamentados en variables cualitativas y cuantitativas previamente registradas desde la etapa de planificación, fue posible diseñar un sistema automatizado que permitiera aplicar al máximo las teorías estadísticas según ciertas variables de mercado, definir una metodología para predecir la demanda de ventas por número de artículos (incluso con la presencia de ofertas); así se logró un nivel de predicción y estructura consistente en el tiempo para los productos de una compañía con estilo de venta directa, que permitiese la programación posterior de una herramienta automatizada para ejecutar proyecciones y simulaciones en tiempo real.

Esta herramienta automatizada presenta un amplio valor en mercados emergentes debido al potencial incremento del mercado por factores como la tasa de crecimiento del grupo que utiliza los productos (según edad, ingresos), competencia tanto en el sector formal como en el informal, y circunstancias generales del entorno, como situación económica, índice de precios al consumidor y cambios en la forma de vida (Allen, 1985).

La ausencia de estabilidad en la demanda es una característica común de los mercados emergentes, por lo cual los buenos pronósticos se convierten en un factor clave para el éxito de la empresa. Las predicciones equivocadas pueden desembocar en inventarios excesivamente grandes, en precios subestimados que resultan muy costosos o, incluso, en la pérdida de ventas producto de la falta de existencias. Por consiguiente, el cálculo de estimaciones en las ventas se debe realizar de una manera rápida y confiable, siendo muy importante para la planificación de las actividades en el futuro que permita desarrollar estrategias para enfrentar un mercado altamente competitivo (Boada, 2011).

Por esta razón, el proyecto derivó en un sistema basado en productos de vida regular con características similares, es decir, aquellos que permanecen a lo largo de ciertos catálogos de ventas precisamente con la intención de disponer de 
información histórica sobre el comportamiento de su línea (Boada, 2013).

\section{Mercadeo vs. administración de la demanda futura}

En toda empresa de consumo masivo, el área de mercadeo establece las estrategias novedosas, nuevos lanzamientos y productos, en función a la creatividad, nuevas tendencias y conocimientos del negocio.

Desde esta perspectiva, las estrategias de marketing se pueden comparar con las estrategias basadas en las situaciones competitivas más habituales en cualquier tipo de industria: los océanos rojos y los océanos azules (Chan-Kim, 1990):

- Los océanos azules simbolizan las ideas de negocio creativas; crean y captan una nueva demanda, rompiendo la disyuntiva del valor o el coste, y alinean todo el sistema en las actividades de una empresa con el propósito de lograr diferenciación.

- En los océanos rojos, la perspectiva tradicional de competitividad en la participación de mercado reta a la competencia, explota la demanda existente en el mercado y alinea todo el sistema en actividades con decisión estratégica.

Un sistema de proyección sobre la demanda futura, como el SPD, permite a la compañía enfocarse en las estrategias competitivas (especialmente aplicadas en el ámbito de los océanos rojos), brindando una mayor libertad de trabajo en consonancia con los océanos azules.

Los estudios de demanda son el mejor medio de conocer y definir la estrategia de mercadotecnia de una empresa; sin embargo, en el ámbito moderno de la globalización, la administración de demanda se ha convertido en el enlace estratégico entre las áreas creativas de mercadeo y las áreas operativas de logística y cadena de suministro.

La finalidad de los departamentos, áreas de administración de la demanda y sistemas de proyección es principalmente cuantificar las estrategias creativas de los departamentos estratégicos, para generar planificaciones logísticas de compras, producción e inventarios futuros que permitan garantizar la disponibilidad del producto en el momento determinado. Así mismo, esta valoración de demanda futura aporta insumos considerables al departamento de finanzas y a la gerencia general en lo relativo a ingresos y márgenes de ganancias futuros, con base en dichos escenarios creativos.

Un sistema de proyección establece estimaciones fundamentadas en el comportamiento histórico de la compañía (a partir de las estrategias de marketing realizadas previamente). No obstante, es muy importante dejar establecido que situaciones macroeconómicas, el desarrollo de la competencia y las estrategias novedosas de marketing (océanos azules) generan nuevos escenarios, los cuales vale la pena profundizar y analizar mediante metodologías adicionales de análisis (Schiffman \& Kanuk, 1991) y percepción del consumidor hasta la decisión real de compra (incluidos factores como la elección del producto, la marca, la elección de la tienda y el método de pago), e incluso lo que ocurre después de la compra (análisis de periodos de incertidumbre, satisfacción, insatisfacción o aun readquisición).

\section{Modelo estadístico de predicción: ¿Es posible predecir escenarios futuros de ventas?}

Para lidiar con el ambiente de marketing y hacer compras, los consumidores entran en un proceso de decisión de compras, definido por Stanton (2007) como la resolución de problemas según las diversas alternativas que ofrece el mercado; sin embargo, en planificaciones masivas en empresas de consumo masivo, el propósito de la administración de la demanda es coordinar y controlar todas las fuentes de demanda, con el fin de poder usar con eficiencia el sistema productivo y entregar el producto a tiempo, logrando un óptimo nivel de servicio y respetando los niveles óptimos de costos (Chase et al., 2005).

En el universo de los pronósticos, estos se pueden clasificar en cuatro tipos básicos: cualitativo, análisis de series de tiempo, relaciones causales y simulación (Lind, 2015).

- Las técnicas cualitativas son subjetivas, y se basan en estimados y opiniones.

- El análisis de series de tiempo utiliza la información relacionada con la demanda pasada para predecir la demanda futura, tomando el tiempo como principal variable explicativa. 
- Por otro lado, el pronóstico causal -utilizado principalmente en este estudio- supone que la demanda se relaciona con diversos factores subyacentes (internos o externos) del ambiente empresarial. Para este tipo de pronósticos la identificación y valoración de las variables causales son fundamentales, e igualmente el uso de manejo de variables mercadotécnicas es primordial para predecir escenarios en empresas con estilo de venta por catálogo como Avon Cosmetics, a diferencia de la venta estilo retail.

- Finalmente, los modelos fundamentados en simulación permiten al encargado del pronóstico valorar y manejar varias "suposiciones" acerca de la condición del pronóstico, aspecto principalmente utilizado en valoración de acciones y activos financieros.

Al utilizar los pronósticos causales, el método de regresión lineal múltiple se hace protagonista, ya que expone los componentes principales de la demanda de productos o servicios: demanda promedio, tendencia, elementos estacionales, cíclicos, variación aleatoria y autocorrelación (Chase et al., 2005).

Con base en esta estructura de formación, se estableció la predicción de escenarios futuros mediante un modelo predictivo compuesto que utilice los beneficios de un modelo de regresión múltiple, con los ajustes automáticos de residuos que proporciona un modelo lineal dinámico bayesiano, estableciendo la estructura básica de la siguiente forma:

Ln(Demanda $)=$ Resultado Modelo de Regresión Múltiple

(Primera parte) + Ajuste Residuo Modelo Lineal

Dinámico Bayesiano (Segunda parte)

donde dicho modelo bayesiano se obtiene con información histórica actualizada hasta tiempo t-1.

\section{Primera parte: modelo de regresión múltiple}

Corresponde a la primera parte del modelo de predicción, es decir, la creación de un modelo de regresión múltiple que se fundamente en variables de mercado y mercadotecnia. Se utilizaron productos con historial relevante ("vida regular"), agrupándolos en categorías con características y usos similares: desodorantes, colonias líquidas, talcos y polvos, champús y acondicionadores, etc.
Esta es la parte fundamental de la predicción, ya que contiene variables explicativas que funcionan según las ofertas de promoción del producto, la publicidad, el tiempo de vida y el número de vendedores o representantes por campaña. Por este motivo, es posible conocer el impacto que proporcionará la alteración de cada variable de mercado en la demanda del número de artículos para cada producto (Webster, 2000; Boada, 2012).

El análisis de regresión múltiple está efectuado de forma que una variable dependiente, Demanda de número de artículos en productos de vida regular, se logra relacionar con dos o más variables independientes, Variables de Mercadotecnia.

$$
\begin{aligned}
& \begin{array}{c}
\text { Demanda artículos vida regular }=\text { función (variables } \\
\text { mercadotecnia) }+ \text { errores. }
\end{array} \\
& Y=X . \beta+\varepsilon \\
& E \text { (Demanda artículos vida regular) }=\text { función (variables } \\
& \text { mercadotecnia) } \\
& E(y)=\beta_{0}+\beta_{1} \cdot X_{1}+\beta_{2} \cdot X_{2}+\ldots+\beta_{p} \cdot X_{p}
\end{aligned}
$$

Si se conocieran los valores $\beta_{0}, \beta_{1}, \beta_{2}, \ldots, \beta_{p}$, se utilizaría la ecuación (1) para calcular el valor medio de " $y$ " dados los valores de las variables de mercadotecnia $\mathrm{x}_{1}, \mathrm{x}_{2}, \ldots, \mathrm{x}_{\mathrm{n}}$. Infortunadamente, esos parámetros se desconocen y se deben determinar a partir de los datos de la muestra; por tal motivo, se calcularon estimadores puntuales de los parámetros anteriormente descritos.

$$
\hat{Y}=b_{0}+b_{1} \cdot x_{1}+b_{2^{2}} \cdot x_{2}+\ldots+b_{p^{\prime}} \cdot x_{p}
$$

en donde

$b_{0}, b_{1}, b_{2}, \ldots, b_{n}$ son los estimados de $\beta_{0}, \beta_{1}, \beta_{2}, \ldots, \beta_{p}$ $\hat{Y}=$ Valor estimado de la demanda del número de artículos para los productos de vida regular.

El método utilizado para deducir la ecuación estimada de regresión múltiple es el "método de mínimos cuadrados", el cual emplea los datos de la muestra para determinar los valores de $b_{0}, b_{1}$, $b_{2}, \ldots, b_{n}$ que minimizan la suma de los cuadrados de las desviaciones entre los valores observados y estimados de la variable dependiente.

Durante el análisis de regresión múltiple, cada coeficiente de regresión $b_{i}$ representa un estimado de la variación en la cantidad demandada de artículos para los productos de vida regular cuando existe un cambio unitario en una variable de mercadotecnia $\mathrm{x}_{\mathrm{i}}$ cuando todas las demás variables independientes se mantienen constantes (Diebold, 1998). 
Se utilizaron las pruebas F-fisher y t-student para determinar la validez de los parámetros que acompañan a las variables independientes, a través de sus estimadores; de la misma forma, se manejó el supuesto de que las variables explicativas debían ser independientes, ya que si presentan un cierto grado de correlación entre ellas, se estaría frente a un problema de multicolinealidad, lo cual denota la incapacidad de separar los efectos individuales de cada variable sobre la cantidad demandada de artículos de vida regular (Dallas, 1998; Boada, 2011, 2013).

\section{Variables de mercadotecnia utilizadas durante la predicción}

Se determinó agrupar productos con características similares y calcular un modelo de regresión para el conjunto como tal, por ejemplo: colonias líquidas, champús y acondicionadores, desodorantes, talcos y polvos, etc.

\section{CAMPAÑA}

Corresponde a la duración del catálogo de ventas. En el caso de Avon Cosmetics, se realizan 18 campañas anualmente, y se registran en el sistema al momento de realizar la estimación. Al seleccionar una campaña, se puede obtener automáticamente información constante inherente a la campaña, como por ejemplo: número de representantes o vendedoras, tasa de inflación, entre otras. A nivel laboral, el proceso de estimación se realiza por campaña; sin embargo, la valoración de las estimaciones se hace por producto, estimando su competencia y "canibalización" interna ${ }^{1}$.

\section{Código deL ARTículo}

Mediante el código del artículo se puede enlazar información constante inherente al producto, que se puede obtener automáticamente mediante otros sistemas internos de la compañía, por ejemplo: nombre del artículo, categoría o familia de productos, precio full y precio de oferta establecido para dicha campaña, tipo de artículo o ponderación del producto dentro de la misma familia, entre otros.

FIGURA 1. Sistema de proyección de la demanda. Módulo de llenado de variables de mercadotecnia

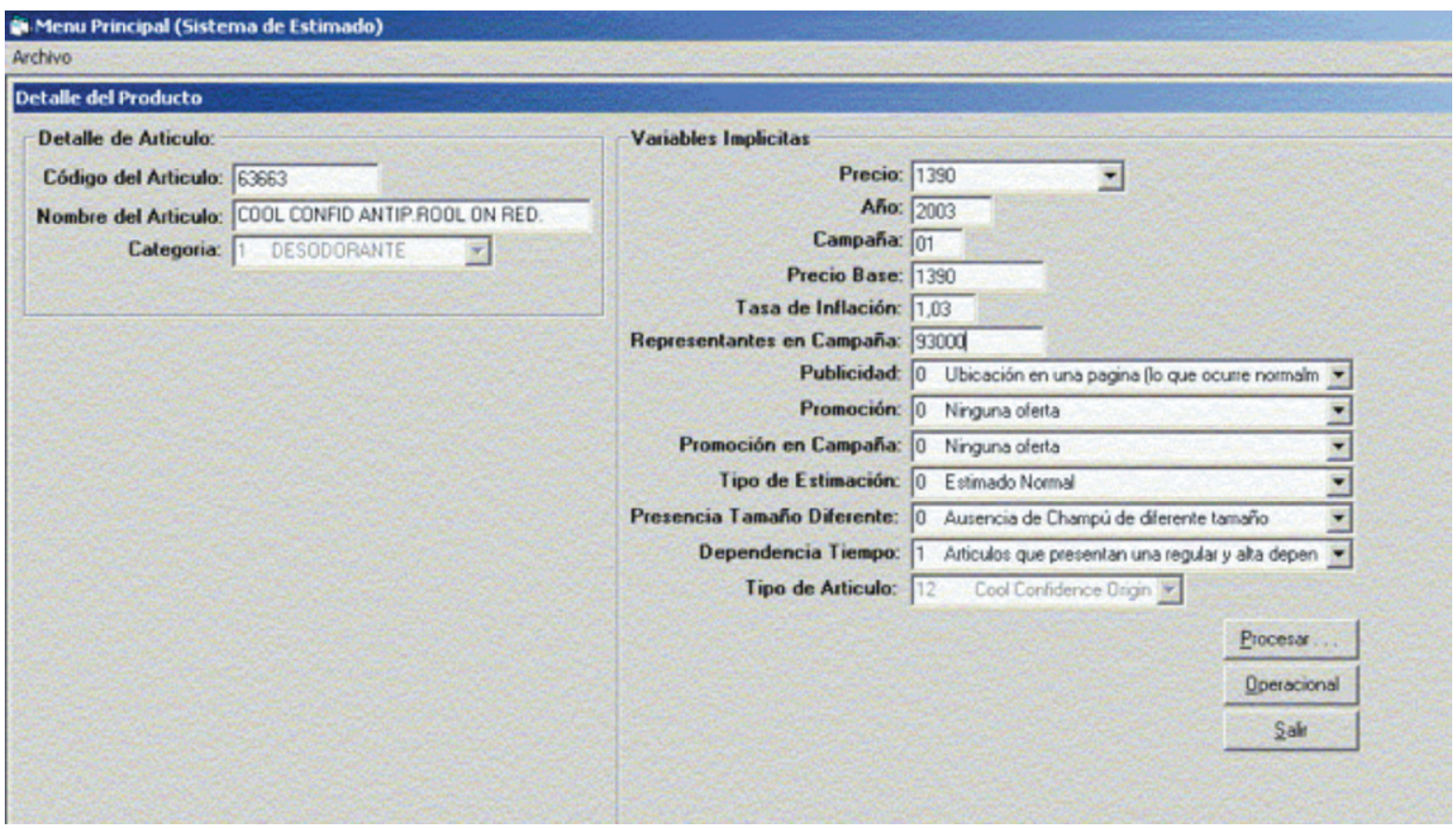

1 Término particularmente acuñado para denotar el debilitamiento del impacto promocional sobre un producto, debido a las múltiples ofertas de productos similares durante el mismo catálogo de ventas.

- 


\section{NÚMERO DE REPRESENTANTES}

Corresponde al número de vendedoras o consejeras que posee la compañía en todo el territorio nacional por cada campaña o catálogo de ventas.

\section{PUBLICIDAd}

Representa una variable cualitativa, la cual es constituida por la ubicación de la foto del producto en estudio en el catálogo de ventas. Entre los indicadores publicitarios tenemos: Una página, Spread, Big Product, entre otros. A pesar de ser una variable cualitativa, se puede cuantificar su impacto impulsador o inhibidor según la técnica estadística de números índices.

\section{PROMOCIÓN}

Es una variable cualitativa que corresponde a las diferentes ofertas promocionales que la empresa coloca al artículo en estudio por campaña. Las características de esta variable se limitan a la data histórica de los productos por estudiar. Si la empresa desea efectuar una oferta que nunca había realizado, el modelo desconocerá directamente la nueva actividad, quedando en el estimador la responsabilidad de enlazarlas según las existentes. Entre las ofertas utilizadas históricamente están los diferentes "porcentajes de descuento", "ambos por", "paquetes", "dos por iguales o diferentes", etc. A pesar de ser una variable cualitativa, es posible cuantificar su impacto impulsador o inhibidor según la técnica estadística de números índices.

Durante el desarrollo de este sistema, se determinó que para lograr un mejor estudio de los artículos era necesario tomar en cuenta ciertos indicadores que no son especificados en la aplicación original de la empresa cliente; de allí surgió la necesidad de revisar y reestructurar manualmente todos los datos suministrados, creando un nuevo esquema (detallado) de indicadores publicitarios y promocionales que derivara en una estructura óptima y robusta de registro continuo de la información en tiempo real similar a un Big data para estimación y simulación de escenarios en tiempo real.

\section{PRECIO}

El precio de venta como variable absoluta presenta una mezcla de otros indicadores, como son: el transcurso del tiempo, la inflación ocurrida históricamente en el país, la variación de los costos de producción, entre otros. El sistema toma en consideración las siguientes características:

1. Relación precio de venta-tasa inflacionaria. Todo producto debe sufrir aumento en su precio de venta de acuerdo con la tasa inflacionaria del país (por campaña de ventas). Si el precio aumenta menos que proporcionalmente respecto al precio inflacionario, la cantidad demandada puede aumentar, sin necesidad de presentar oferta alguna; lo mismo ocurriría durante las campañas en las que el precio permanece constante, mientras que la inflación continúa su rumbo.

2. Es necesaria la presencia de una relación entre el precio de oferta y el precio normal (sin oferta), ya que indicaría el peso adicional que sufriría la cantidad demandada bajo ese indicador promocional.

\section{CATEGORÍA O FAMILIA DE PRODUCTOS}

Expone una agrupación de productos cuyas características físicas o de uso son similares. Esta agrupación es ideal para identificar patrones de comportamiento, y por ende lograr predicciones más efectivas; por ejemplo, familias de desodorantes, champús, lociones, entre otras.

\section{TIPO DE ARTí́CULO}

Mediante esta variable se cuantifica el "peso" aproximado que posee cada producto respecto a un artículo base. Pese a que los modelos agrupan productos similares, el sistema predice individualmente por artículo; esto debido a que el nivel de ventas es netamente particular e independiente. El sistema presenta la posibilidad de añadir nuevos artículos (siempre y cuando pertenezcan a los que poseen modelo elaborado), únicamente colocando como información previa la posible demanda promedio por campaña.

Esta variable se utiliza como información inicial de predicción, puesto que posteriormente el sistema de predicción ajustará de forma automática su nivel de ventas según las últimas campañas de historia.

\section{Presencia de tAMAÑo DifERENTE}

Mediante esta variable, el sistema de predicción considera la posible variación en la demanda 
si el producto es ofertado adicionalmente en tamaños diferentes para determinada campaña (actualmente disponible en las colonias líquidas y champús-acondicionadores); por ejemplo, colonia de igual fragancia en $100 \mathrm{ml}$ y $15 \mathrm{ml}$.

\section{TIPO DE ESTIMACIÓN}

Esta es una variable subjetiva, la cual se encontrará a juicio del estimador. De esta manera, el sistema podrá realizar estimaciones controladas en exceso o en defecto. Posee tres (3) niveles de sobrestimación y dos (2) de baja estimación.

Esta característica es de suma importancia, en razón de que permite la actuación sistemática del estimador al momento de surgir una situación no contemplada en el sistema (sin necesidad de esperar que el modelo se actualice). Por ejemplo: cambios extremos en la situación del país, contingencias "especiales", ofertas novedosas.

Entre dichas ofertas, se puede resaltar:

a) cuando se obsequia un producto con la compra de otro más económico;

b) cuando dos o tres artículos son ofertados a un precio menor que el individual, etc.

El proyecto utilizó principalmente el software S-Plus 4.5, complementado con el SPSS 7.5 y el Microsoft Excel, entre otros.

\section{Cómo cuantificar variables cualitativas}

Para dicho proyecto, se estableció un procedimiento de cálculo con el fin de ponderar el impacto potenciador o inhibidor de cada término de una variable cualitativa sobre el comportamiento de la demanda de una familia de productos.

Para estas variables fue necesario crear un nivel de cuantificación del impacto de cada característica dentro de cada familia de productos. Por tal motivo se procedió a utilizar el procedimiento de ponderación mediante números índice simple, en donde se maneja una transformación de escala que manipula los valores para asegurar la compatibilidad con otras escalas (Webster, 2000). Los componentes para el cálculo de los índices fueron:

$\mathrm{PBD}_{\text {Ausencia }}$ : Promedio Base de la Demanda de productos cuando hay Ausencia del término de la variable cualitativa, calculado para cada familia de productos específicos, el cual corresponde al indicador más básico y común de la variable cualitativa de un producto perteneciente a cualquier familia dentro del catálogo.

$\Sigma($ Cantidad Demanda de los
$P B D_{\text {Ausencia }}:$
$\begin{gathered}\text { artículos cuando hay Ausencia } \\ \text { pertenecientes a cada familia) }\end{gathered}$
$\begin{gathered}\text { Número de ocasiones en las que existieron } \\ \text { productos con ausencia de la Var } \\ \text { Cualitativa para dicha familia }\end{gathered}$

$\mathrm{PD}_{\text {Característica (i) }}$ : Promedio de la demanda, calculado para cada familia de productos específicos según cada característica de la variable cualitativa (i), registrada en su base histórica de ventas.

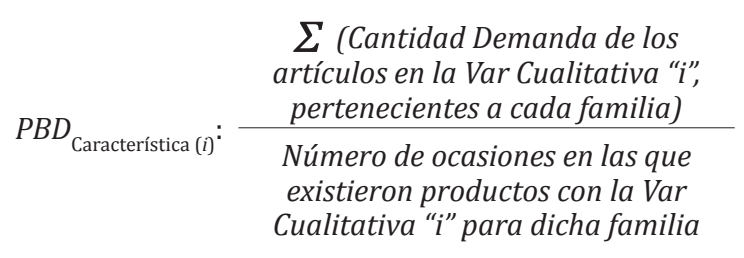

Luego, los indicadores de impacto cualitativos (IIC) se calculan para cada promedio de la demanda (PD), en comparación de su diferencia porcentual con el único promedio base de la demanda (PBD); esto suavizado a nivel logarítmico para mantener la estabilidad lineal del modelo original semi - log.

En este sentido, el indicador de impacto cualitativo (IIC) potenciador o inhibidor para cada característica "i", de cada variable cualitativa "j" se calcularía de la siguiente manera:

$$
I I C_{(i, j)}=\left(\frac{\operatorname{In}\left(P D_{\text {Caracteristica( } i, j)}\right)}{\operatorname{In}\left(P B D_{\text {Ausencia(j) }}\right)}-1\right) \cdot 100
$$

De esta manera se obtuvieron tantos indicadores de una variable cualitativa, como características se presentaron en la data histórica inherente a la familia de productos.

Análogamente, si una oferta que no aparece en la tabla de promociones comienza a ser repetitiva en los productos estudiados, un usuario maestro podrá calcular su efecto e introducirlo en la lista de indicadores, para que pueda ser utilizado posteriormente por los estimadores.

\section{Ejemplo del modelo estadístico de regresión múltiple desarrollado (MRM)}

Tomando estas variables de entrada fue posible realizar diversas pruebas en familias de 
productos de Avon Cosmetics, logrando resultados interesantes en cuanto a ajuste, consistencia y robustez, usando el método para la inclusión de variables denominado backward o eliminación descendente. En los estudios manejados, se lograron niveles de ajuste en $\mathrm{R}^{2}$ entre $70 \%$ y $85 \%$, con una mínima multicolinealidad entre las variables y un comportamiento estadístico de residuos estadísticamente aceptables, en cuanto a aleatoriedad y normalidad, y con amplia estabilidad en la varianza (Boada, 2013).

A continuación se presentan los resultados de una de las familias analizadas para esta compañía multinacional.

\section{FAMILIA: LOCIONES PARA DAMAS}

Definición: corresponde a todos los productos inherentes a lociones líquidas y cremosas de manos y cuerpo, dirigida para el público femenino.

Se realizó una corrida a través de la aplicación estadística S-plus $₫$ para determinar la ecuación de regresión en la familia de productos Lociones Damas; los resultados obtenidos (a nivel de coeficientes $\beta$ ), indicaron impactos significativos sobre la transformación logarítmica de la demanda por SKU en cada campaña de ventas, como se observa en el Cuadro 1.

La ecuación de regresión multivariada para esta familia en particular con la información procesada a través de la aplicación estadística S-Plus ${ }^{\circledR}$, fue la siguiente:

$$
\begin{aligned}
& \operatorname{Ln}(\text { Demanda })=453,89170000-0,13596080 * V 18+ \\
& 0,03887495 * V 22+1,22522600 * V 25+0,00046793 \\
& \quad * V 26+0,00132950 * V 27+0,00842663 * V 28- \\
& \quad 0,2242180 * V 30
\end{aligned}
$$

Con esta fórmula de regresión multivariada se puede lograr la predicción inicial del modelo estadístico predictivo, en función de los impactos potenciadores e inhibidores de las variables de mercadeo.

Al verificar tanto el estadístico " $F$ ", como los valores " $\mathrm{p}$ " resultantes de las pruebas " $\mathrm{t}$ ", se obtuvo que todos los coeficientes estimados para las variables tomadas en consideración son significativamente diferentes de cero, con un coeficiente $\mathrm{R}^{2}$ de determinación múltiple de $81,69 \%$, lo que indica un alto porcentaje de variabilidad explicado por el modelo que tuvo un poco más de 1.500 datos.

\section{COEFFICIENTS:}

\begin{tabular}{crrrr}
\hline & VALUE STD. & \multicolumn{1}{l}{ ERROR } & \multicolumn{1}{l}{ TVALUE } & PR(>|T|) \\
\hline (Intercept) & 453.8917 & 48.7160 & 9.3171 & 0.0000 \\
\hline V18 & -0.1360 & 0.0673 & -2.0211 & 0.0435 \\
\hline V22 & 0.0389 & 0.0009 & 41.7599 & 0.0000 \\
\hline V25 & 1.2252 & 0.1032 & 11.8731 & 0.0000 \\
\hline V26 & 0.0005 & 0.0002 & 2.3624 & 0.0183 \\
\hline V27 & 0.0013 & 0.0000 & 29.7885 & 0.0000 \\
\hline V28 & 0.0084 & 0.0009 & 9.5151 & 0.0000 \\
\hline V30 & -0.2242 & 0.0243 & -9.2431 & 0.0000 \\
\hline
\end{tabular}

Residual standard error: 0,7114 on 1099 degrees of freedom

Multiple R-Squared: 0,8169

F-statistic: 700,3 on 7 and 1099 degrees of freedom, the p-value is o

Así mismo, los análisis de residuos cumplen los aspectos de normalidad y aleatoriedad de residuos.

\section{Segunda parte: modelo bayesiano}

\begin{tabular}{|c|c|c|c|}
\hline NOMBRE DE LA VARIABLE & CÓDIGO & COEFICIENTE & $\begin{array}{l}\text { INFLUENCIA SOBRE } \\
\text { EL LN(DEMANDA) }\end{array}$ \\
\hline Constante & & 453,89170000 & \\
\hline Variable binaria tamaño diferente & $V_{18}$ & $-0,13596080$ & Inhibidora \\
\hline Indicador del tipo de artículo & $V_{22}$ & 0,03887495 & Potenciadora \\
\hline Indicador de precio & $V_{25}$ & 1,22522600 & Potenciadora \\
\hline Variable exposición & V26 & 0,00046793 & Potenciadora \\
\hline Variable promoción & V27 & 0,00132950 & Potenciadora \\
\hline Variable promoción canibalizadora & $V_{2} 8$ & 0,00842663 & Potenciadora \\
\hline Variable fecha & $V_{30}$ & $-0,22421800$ & Inhibidora \\
\hline
\end{tabular}

Los resultados obtenidos en el apartado anterior mediante los modelos de regresión

CUADRO 1. Coeficientes estimados para el modelo de familia Lociones para damas 
FIGURA 2. Gráficos de residuos del MRM para Lociones para damas
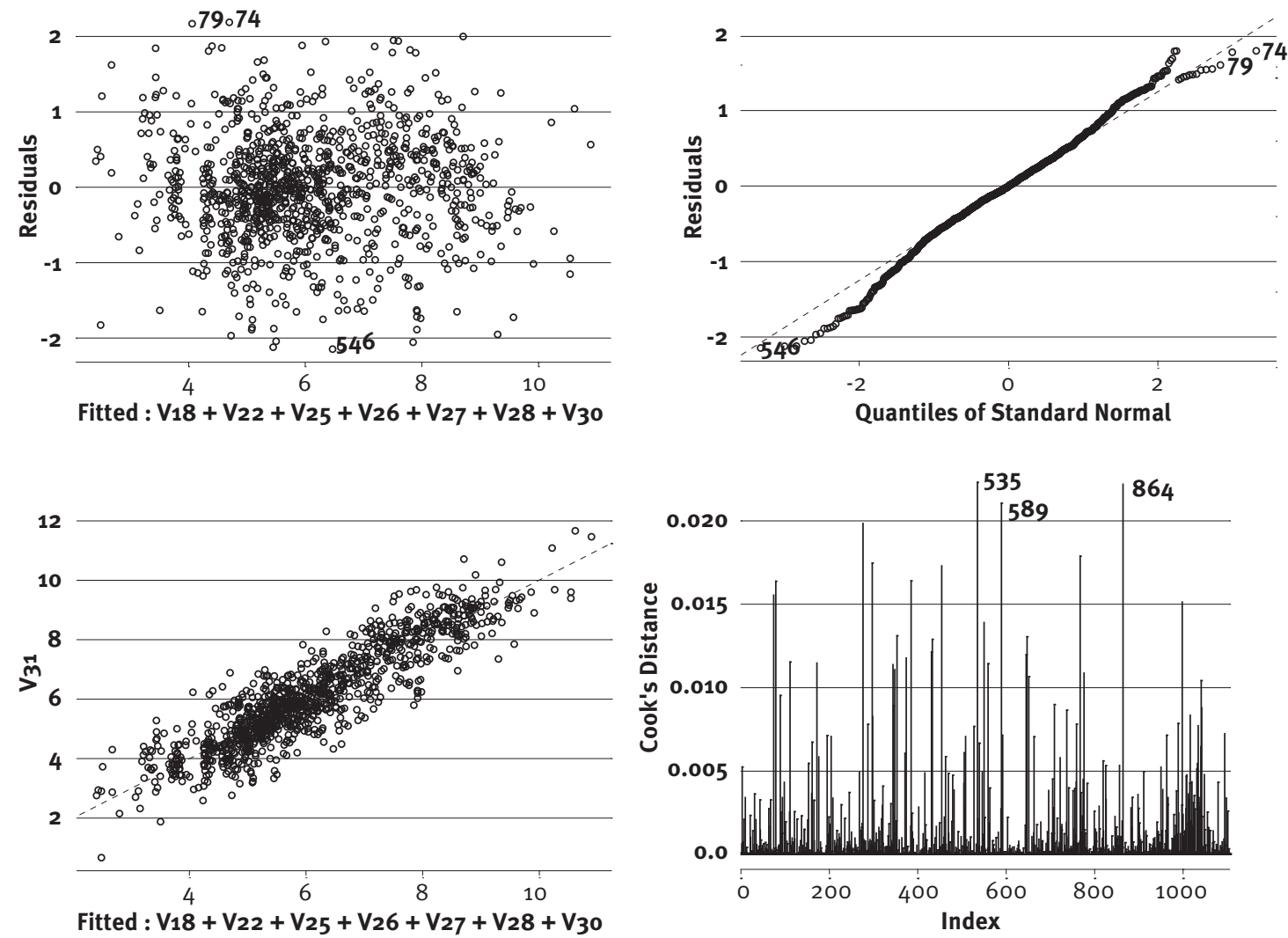

Fuente: resultados del proyecto.

multivariada presentan una estimación fundamentada en el uso de las variables de mercadotecnia según las estrategias de marketing; sin embargo, según Peter (2006), es posible que el comportamiento consecuente de la demanda del producto (en lo relativo a su tendencia) sufra alteraciones en el tiempo, ante escenarios como los siguientes:

- Introducción de un nuevo competidor, tanto en el ámbito de producto como de empresas.

- Variación en el número y duración de las campañas de ventas.

- Modificación extrema de la situación del país.

- Nuevo nivel de ventas, originado por el rediseño del producto.

- Alteración excesiva en el precio de venta.

La estadística bayesiana provee un marco teórico ideal para el modelaje de datos, ya que permite tratar los problemas con una claridad axiomática y flexibilización en forma simultánea, lo cual hace posible desarrollar inferencias coherentes, para establecer un procedimiento robusto, estable y consecuente que facilite solidificar la programación en un sistema de proyección de la demanda (SPD).

Dicha parte bayesiana es ejecutada mayormente - por las limitaciones que presenta el modelo de regresión múltiple- en lo relacionado con la demanda de artículos, la cual permanece constante en los modelos de regresión (Pericchi, 2002). Estas causas pueden producir una alteración en el nivel de demanda según el tipo de artículo. A través del modelo dinámico bayesiano se puede determinar el efecto producido por cualquiera de estas causas que no toma originalmente el modelo de regresión múltiple, esto con pocos catálogos (campañas) de historia de ventas, a fin de ajustar la evolución de la cantidad demandada en el tiempo (West \& Harrison, 1989). De la misma forma, toma en consideración cambios continuamente sutiles, es decir, puede adaptarse automáticamente a modificaciones leves en los niveles de venta (Boada, 2011). 
A pesar de que dichos modelos de regresión múltiple se elaboran en categorías (familias) con características similares (desodorantes, champús, colonias líquidas, etc.), sus ajustes se efectúan de manera individual por producto inmediatamente después de obtener la información de la demanda real; por este motivo la capacidad de reacción y ajuste es superior a la de cualquier estimador.

\section{Modelo lineal dinámico de orden 1}

Este modelo corresponde a la segunda etapa del modelo estadístico predictivo, realizado directamente en los residuos anteriormente especificados, logrando una distribución predictiva que evolucionará en el tiempo, y cuyos niveles serán añadidos al resultado de predicción futura " $t+1$ ", proporcionado por el modelo de regresión múltiple.

\section{TEOREMA 1}

Para cada instante de tiempo $t=1,2,3, \ldots$, consideremos las siguientes ecuaciones de observación y sistema (West \& Harrison, 1989; Pericchi, 2002):

\section{Ecuación de Observaciones}

$\mathrm{y}_{\mathrm{t}}=\mu_{\mathrm{t}}+\mathrm{v}_{\mathrm{t}}$, donde $\mathrm{v}_{\mathrm{t}} \sim \mathrm{N}\left(0, \mathrm{~V}_{\mathrm{t}}\right)$

\section{Ecuación Sistema}

$$
\mu_{t}=\mu_{t-1}+\omega_{t} \text {, donde } \omega_{t} \sim N\left(0, W_{t}\right)
$$

Tomando como información inicial $\Pi\left(\mu_{0} \mid \mathrm{D}_{0}\right) \sim$ $\mathrm{N}\left(\mathrm{m}_{0}, \mathrm{C}_{0}\right)$, donde mantendremos los supuestos de aleatoriedad y normalidad de residuos del modelo de regresión múltiple, entonces $\mathrm{m}_{0}=0, \mathrm{y} \mathrm{C}_{0}=1$ (Boada, 2002).

Este componente bayesiano genera una predicción ajustada por nuevo nivel de demanda para cada artículo, calculado a través de un aprendizaje a priori según los errores manejados entre la predicción del modelo de regresión múltiple y el logaritmo de la demanda (luego de haber ocurrido la venta).

Este nuevo nivel de demanda para cada artículo crea un nivel ajustable automáticamente en el tiempo " $t$ ", que mantendrá la consistencia del modelo de regresión múltiple en productos que incrementen o disminuyan su nivel de demanda a medida que evolucionan en el tiempo.

Así mismo, en el caso eventual de que un determinado producto comience a elevar (o

FIGURA 3. Gráfico de predicción arrojado por el sistema de proyección de la demanda (SPD) para un producto particular de Avon Cosmetics

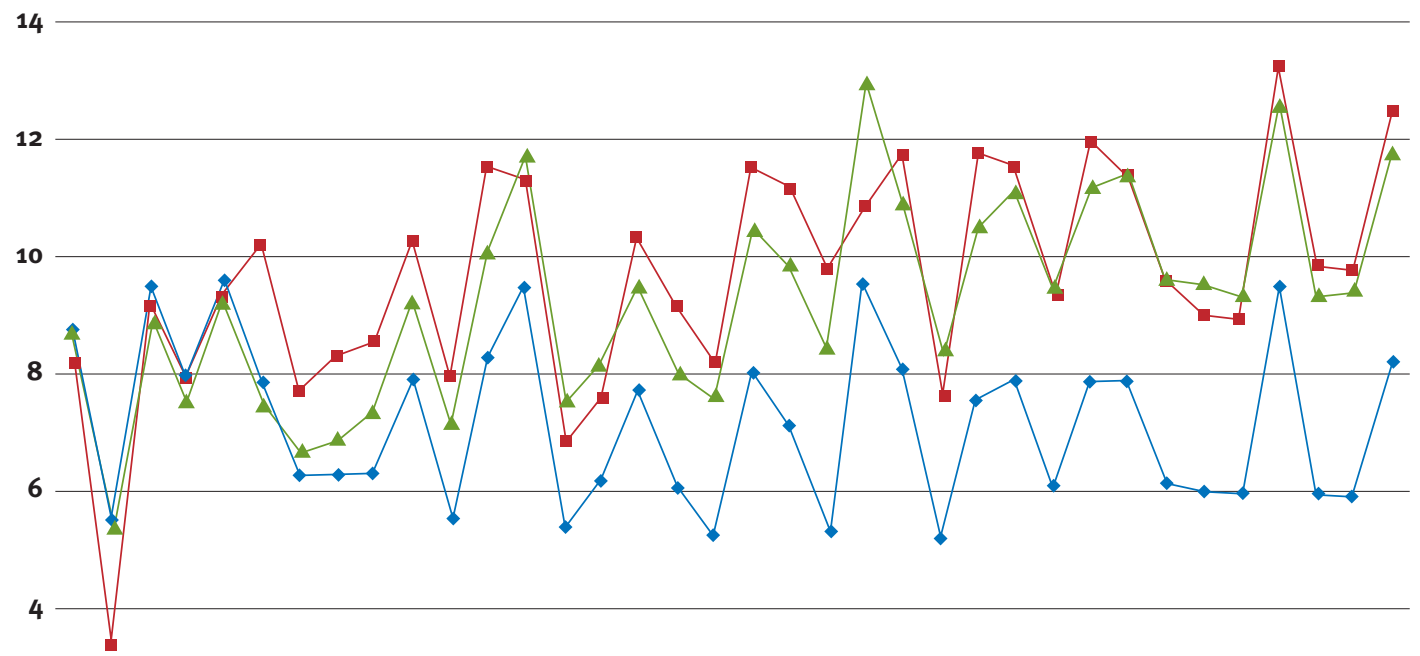

2

o $122 \quad 3 \quad 4 \quad 5 \quad 6 \quad 7 \quad 8 \quad 9 \quad 101112131415161718192021222324252627282930313233 \quad 343536$

$\rightarrow$ Estimación MRM $\rightarrow \operatorname{In}($ Demanda $) \rightarrow$ Modelo Estadistico Final 
disminuir) paulatinamente su nivel de demanda en los últimos catálogos de ventas, producto de situaciones macroeconómicas o por el desempeño de competencias no contempladas en el modelo original de regresión, se aprecia una disparidad entre el comportamiento de la demanda real y las predicciones del modelo de regresión múltiple.

\section{Aplicación automatizada: sistema de proyección de la demanda (SPD)}

El proyecto de investigación inicia desde el año 2000, con la cooperación conjunta entre la Universidad Simón Bolívar y Avon Cosmetics de Venezuela, en búsqueda de un procedimiento estandarizado que permitiese predecir escenarios futuros de ventas, fundamentado en el comportamiento histórico de la compañía, y que facilitase el desarrollo y establecimiento de un sistema estadístico de predicción de demanda, denominado SPD, como aplicación automatizada diseñada con la intención de proporcionar una herramienta adicional al planificador de la demanda. Se buscaba igualmente que esta aplicación, unida a su propia experiencia como planificador, le permitiera obtener con menor esfuerzo un mayor nivel de certeza al momento de predecir la cantidad demandada por producto. Originalmente, el proyecto se gestó según las disposiciones particulares de las empresas con estilo de venta directa, con ecuaciones específicas de Avon Cosmetics para cada país; sin embargo, el patrón detectado puede extenderse a compañías similares, creando modelos estadísticos predictivos ajustados a la información manejada.

El sistema de proyección de la demanda presenta como columna vertebral un modelo estadístico de predicción desarrollado por especialistas en el área, sustentado en la metodología de regresión múltiple con variables de mercado y la técnica de actualización automática del modelo lineal dinámico bayesiano.

Mediante la aplicación automatizada, se muestra la historia del producto, detallando sus

FIGURA 4. Sistema de proyección de la demanda. Consulta de información histórica

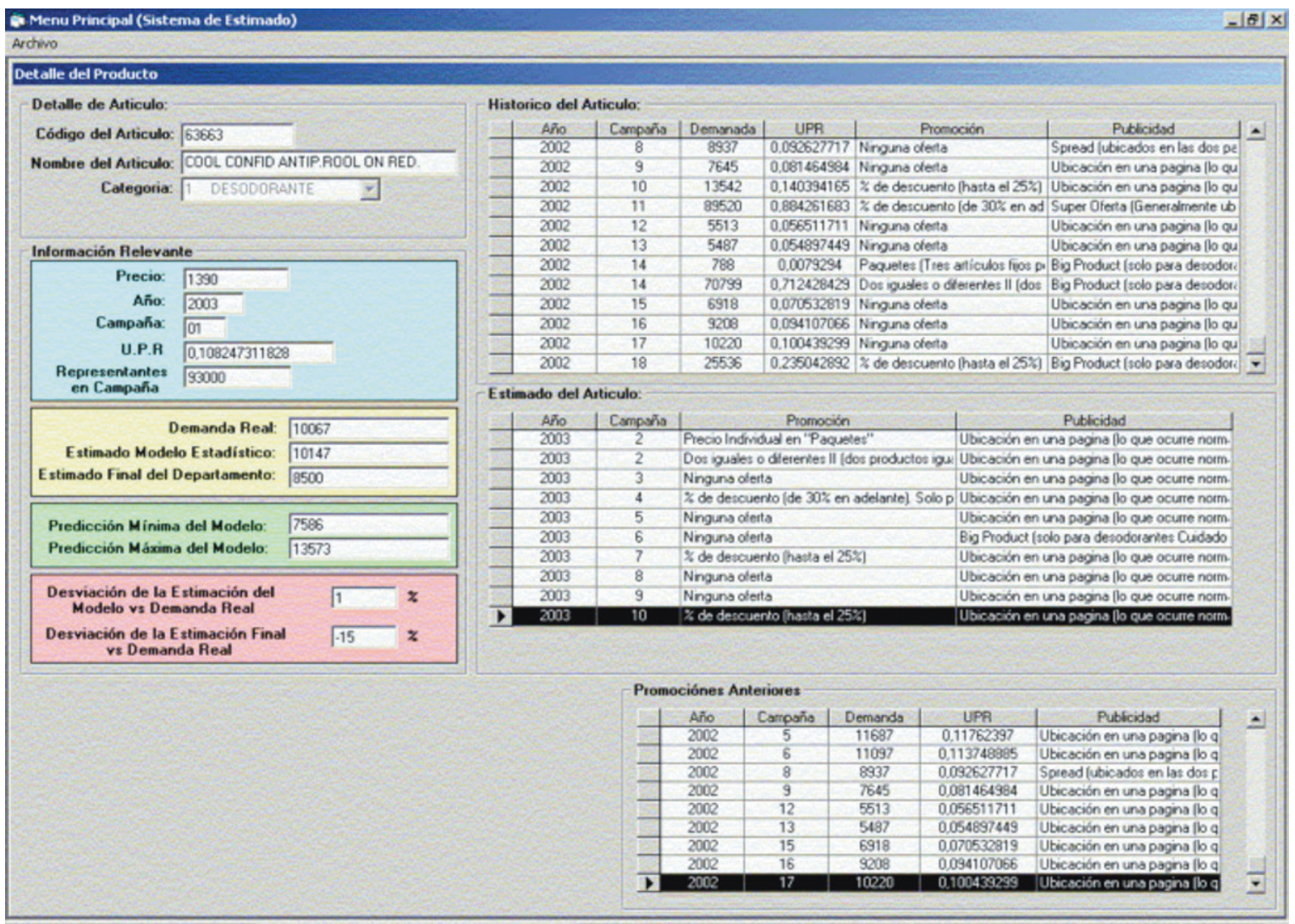


FIGURA 5. Sistema de proyección de la demanda. Consulta de costos y márgenes de ganancia

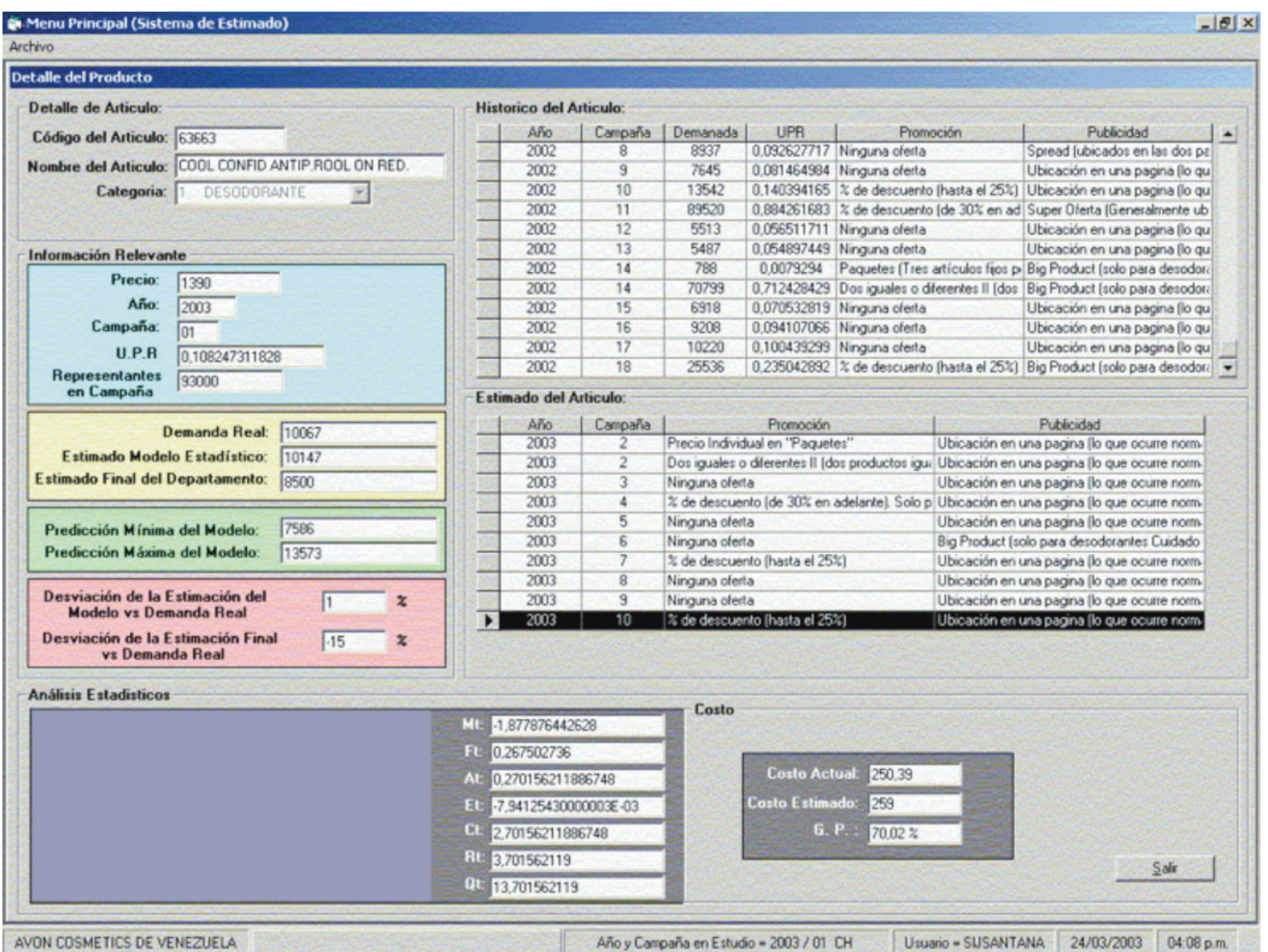

promociones (ofertas) y publicidades (ubicación en el catálogo) por campaña; además, una opción automática de filtraje de información según su promoción, en donde el estimador podrá observar las campañas (demanda y UPR) en las que el artículo presentó el mismo tipo de oferta. Enseña el porcentaje de error (por exceso y defecto) tanto del modelo estadístico como del departamento de estimados (Boada, 2000).

Además de proponer un estimado, el modelo estadístico de predicción establece un intervalo de predicción, en el que, a través de bandas de tolerancia (intervalos de confianza), el estimador podrá colocar su estimado final. Dichos intervalos son adaptables según el error acumulado entre el modelo estadístico y la demanda real final: si el error es bajo, la amplitud de los intervalos será reducida.

Presenta la opción de visualizar los costos estimados y actuales de cada producto por campaña, así como su margen de ganancia (Gross Profit, GP).
FIGURA 6. Sistema de proyección de la demanda. Módulo de enlace entre códigos viejos y códigos nuevos de un mismo producto

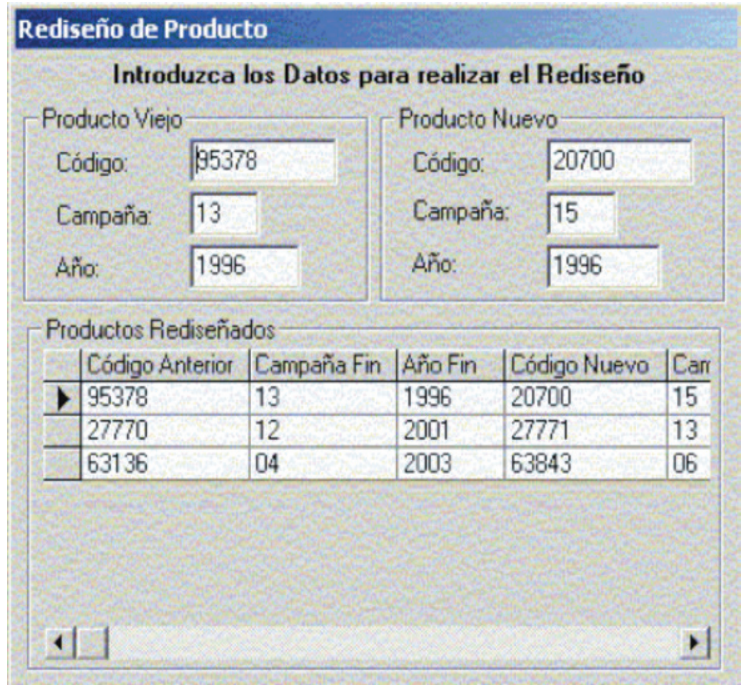

Fuente: resultados del proyecto. 
Uno de los inconvenientes encontrados en el proyecto tuvo que ver con los productos que presentan variaciones menores, tipo de envase o etiqueta, lo cual deriva en un cambio de código y, por ende, en una pérdida completa de su historial de ventas. Para contrarrestarlo se generó un módulo para efectuar un rediseño que permite la opción de "rediseño del producto", con el fin de vincular el nuevo código con el antiguo, y así mantener la historia correspondiente a este último.

De la misma forma, cuando se presenta el lanzamiento de un nuevo producto que no se pueda acoplar directamente con otro, existe la opción de "inicio del producto", donde el estimador coloca el promedio de artículos que piensa vender por campaña y ajusta el sistema para iniciar la predicción según esa premisa (si el producto manifiesta otro nivel de ventas, el modelo se ajustará automáticamente al tomar la demanda real) (Véase figura 7).

Uno de los principales retos de la aplicación automatizada representó la interfaz directa que debe poseer el SPD con los sistemas de información de mercadeo (SIM), la cual consolida el conjunto de procedimientos y métodos para la

FIGURA 7. Sistema de proyección de la demanda. Módulo de inicio de producto nuevo

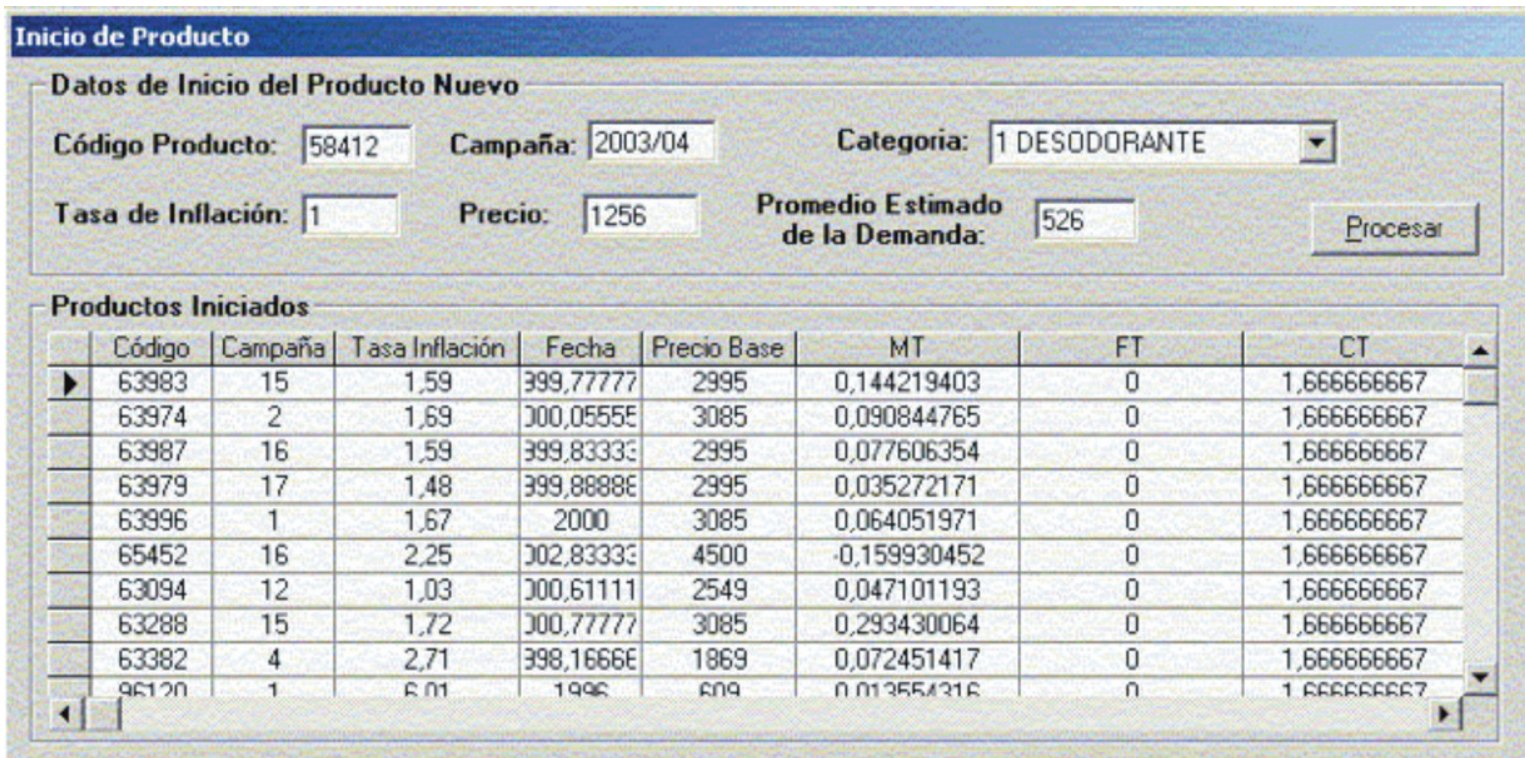

Fuente: resultados del proyecto.

FIGURA 8. Flujograma de alimentación del sistema de proyección de la demanda con los programas corporativos de Avon Cosmetics

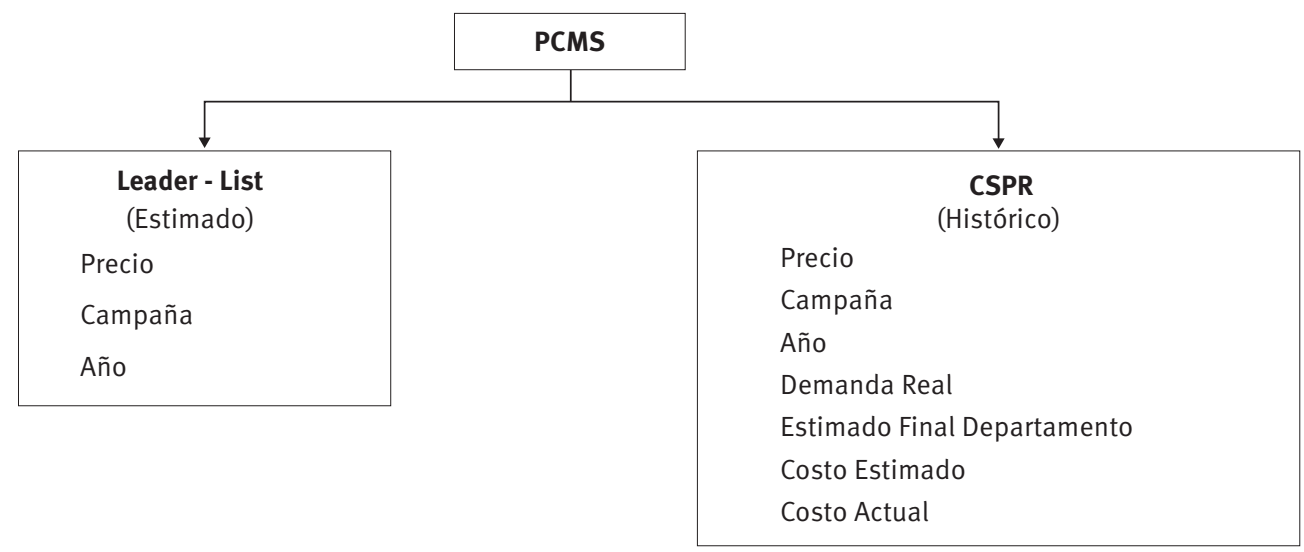


recopilación, análisis y presentación planeados y periódicos de información para su uso en la toma de decisiones de mercadotecnia, en todas sus etapas (Prieto, 2013), y aporta la siguiente información: (Véase figura 8).

Las predicciones son realizadas con base en una tasa de inflación y número de vendedoras estimadas; sin embargo, la aplicación automatizada actualiza dicha información en el momento, a fin de mantener comunicación directa entre todos los departamentos asociados.

El sistema de predicción realiza copias directas de los sistemas primarios de la compañía, esto debido a que se trata de una herramienta de simulación de posibles escenarios futuros, fundamentados según las planificaciones realizadas previamente por los departamentos estratégicos de mercadeo y ventas. Los sistemas Leader List y PCMS corresponden a los sistemas de información de mercadeo (SIM) de la empresa Avon Cosmetics de Venezuela. (Véase figura 9).

Esta forma particular de enlazar genera diversas rutas en las bases de datos (estimado e histórico), produciendo una adaptabilidad estupenda entre el sistema de proyección de la demanda y cualquier sistema maestro de información de la empresa; además, presenta un tiempo definitivamente corto de formulación, cálculo, búsqueda y almacenamiento de la data trabajada.

Así mismo, el sistema puede estimar los productos bajo doble o triple oferta, debido a que realiza distinciones según los precios que proporcione Leader List; de esta manera, se puede predecir en un artículo tanto las ofertas como los precios individuales en la misma campaña de ventas. Para estos casos, el indicador o ID de identificación se encuentra determinado por el código de venta + la campaña de ventas + el precio del producto.

A pesar de que el modelo estadístico se actualiza continuamente de manera automática, se puede predecir independientemente de las campañas cargadas; la diferencia radicaría en que la precisión de la estimación mejorará mientras se aproxime la campaña estimada (las estimaciones preliminares serán actualizadas automáticamente a medida que se aproxime la etapa de revisado y final). De la misma forma, pueden ejecutarse ajustes posteriores en las estimaciones bajo versiones operacionales, observando así la modificación de los estimados según resultados obtenidos en tendencias.

De la misma forma, pueden ejecutarse ajustes posteriores en las estimaciones bajo versiones operacionales, observando así la modificación

FIGURA 9. Flujograma de trabajo, comunicación e interfaz con el sistema de proyección de la demanda

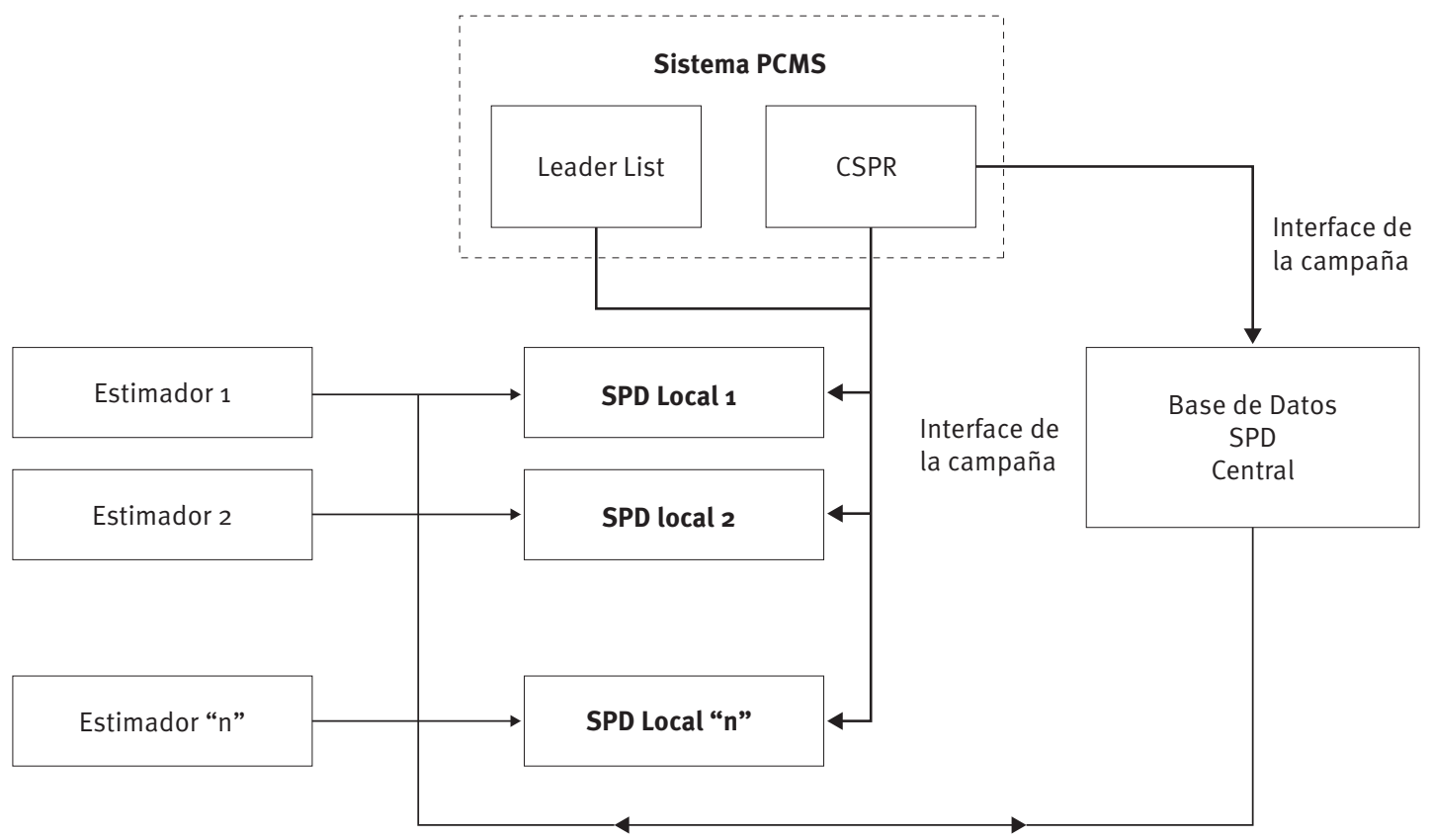


de los estimados según resultados obtenidos en tendencias.

\section{Factibilidad de la automatización de los modelos estadísticos predictivos}

El desarrollo de la primera y la segunda etapa del modelo estadístico se encuentra inmerso en la programación del sistema, de forma transparente para el usuario final, adaptando las estimaciones según los nuevos niveles de tendencia que experimente cada producto en el tiempo.

Finalmente, aparte del fundamento estadístico subyacente en el modelaje utilizado, que se apoya en variables de mercado, el sistema de proyección de la demanda expone al usuario otros aspectos que potencian la aplicación:

Gráficos por producto: de esta forma, el estimador puede observar de forma gráfica la continuidad del producto (demanda real, estimado del modelo estadístico y estimado departamento) tanto del año en curso como del anterior. (Véase figura 10).

En función de los adelantos efectuados en el sistema de imágenes digitalizadas, el estimador puede observar la apariencia que tendrá el producto en el futuro folleto de ventas. (Véase figura 11).

Finalmente, el sistema de proyección de la demanda (SPD) también presenta la opción de compilar productos con variables equivalentes; caso labiales, esmaltes, desodorantes, etc.

\section{Discusión de resultados}

Se debe tener siempre presente que es prácticamente imposible hacer un pronóstico perfecto.

FIGURA 10. Sistema de proyección de la demanda. Módulo de consulta de modelos predictivos

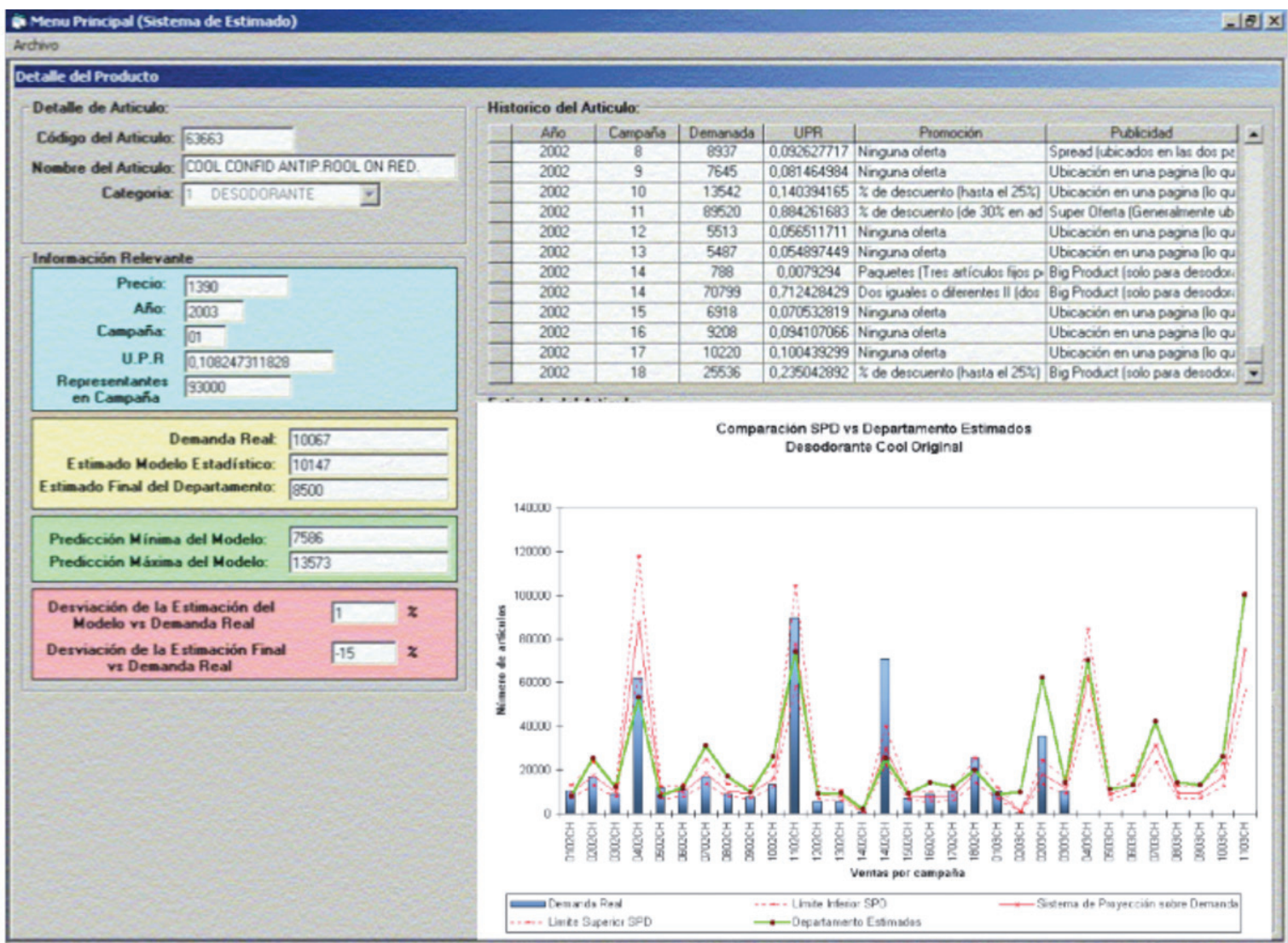


FIGURA 11. Sistema de proyección de la demanda. Módulo de consulta de modelos estadísticos predictivos con imagen del producto

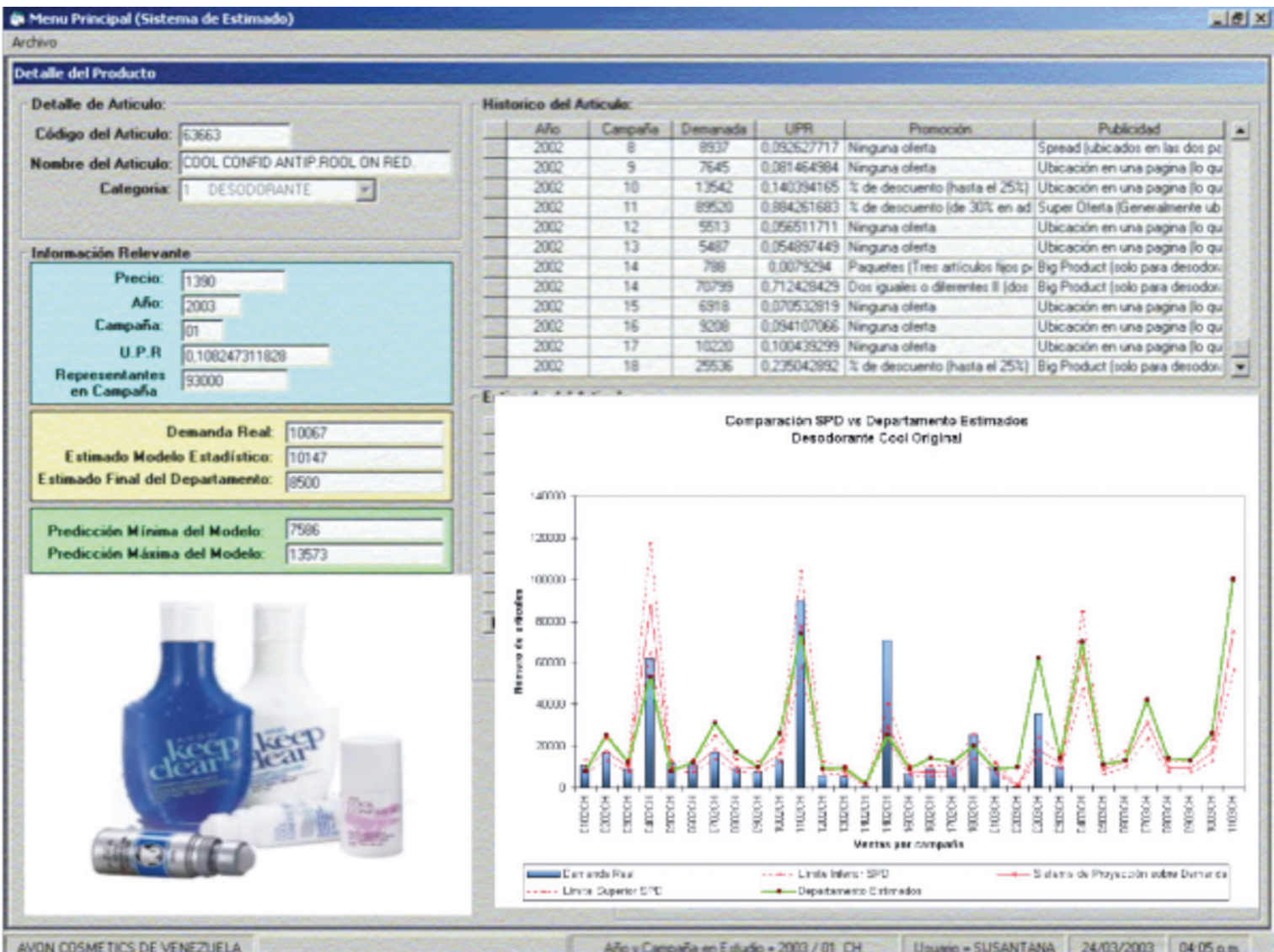

Fuente: resultados del proyecto.

El contexto de los negocios contiene muchos factores que no se pueden prever con certeza. Por tanto, es mucho más importante que, en lugar de pretender un pronóstico perfecto, se imponga la práctica de revisar constantemente los pronósticos y se aprenda a vivir con pronósticos inexactos (Chase et al., 2005). Lo anterior no significa que no se deba intentar mejorar el modelo o la metodología de pronósticos, sino que es preciso tratar de encontrar y emplear el método más conveniente para que los pronósticos sean lo más razonables posible, y con estructuras y procedimientos estables, que puedan ser considerados en una herramienta automatizada de pronóstico.

En este sentido, el sistema de proyección de la demanda se aplica como una herramienta de simulación, en donde al introducir las variables causales se puede obtener una estimación matemática de los impactos potenciadores e inhibidores de la demanda, en tiempos más reducidos que si se aplica el análisis manual de información histórica.

Adicional a la herramienta de simulación fundamentada en el modelo estadístico descrito antes, el sistema presenta un módulo de consulta de información histórica y estimada de código de venta del producto, a fin de facilitar al usuario la búsqueda, consulta de las variables e imagen del mismo en el catálogo, todo esto con miras a optimizar la estimación final del concepto a nivel de SKU.

La herramienta automatizada SPD se creó con la intención de mejorar continuamente el proceso de estimación en las empresas con estilo de venta directa o venta por catálogo, gracias al uso de una aplicación que brinde una información histórica sólida, que unifique y se alimente de otros departamentos, a fin de que el usuario final pueda simular escenarios directamente desde su ordenador, manejando toda la información disponible 
de la empresa. Los modelos estadísticos predictivos presentan un valor agregado al ofrecer al estimador o planificador de la demanda la opción de simular matemáticamente escenarios, brindando un método adicional para su valoración posterior.

En el caso de Avon Cosmetics, las estimaciones presentan tres (3) etapas de valoración de unidades estimadas por producto para cada catálogo futuro: etapa preliminar, etapa de revisado y etapa final. Durante estas etapas el estimador puede iniciar desde una simple simulación matemática, brindada por un modelo estadístico, hasta una valoración más detallada según características históricas similares o aspectos novedosos que pueden hacer fluctuar los estimados más allá de una valoración matemática.

\section{Conclusiones}

Establecer las condiciones que pueden incidir sobre un aspecto tan sensible como la demanda de un determinado producto es un trabajo de gran complejidad. Cabe destacar que se habla de posible asociación de impacto y no de causalidad directa; sin embargo los esfuerzos realizados valen la pena en función de lo que se desea hacer con la información generada por los análisis propuestos, y en el campo de las predicciones la fase de diseño de los análisis es la que tiene la mayor importancia, dado que una decisión mal tomada en esta etapa trae como consecuencia la pérdida de recursos, tiempo, e incluso se puede incurrir en planificaciones basadas en información errónea. Este es el punto de partida de toda predicción: el diseño y la adaptación de las técnicas estadísticas adecuadas, con la finalidad de lograr los objetivos determinados.

El estimador o planificador de la demanda en empresas con estilo de venta directa debe ser un empleado con diversos conocimientos tanto en el área cuantitativa como cualitativa, con marketing, ventas y cadena de suministros (supply chain). En otras palabras, un estimador tiene que ser un profesional integral en todas las áreas de la organización, ya que sus funciones y labores cotidianas radican en aspectos manejados por diferentes áreas de la organización.

El sector de estimados y tendencias es un área que se encuentra inmersa entre los dos canales principales de una compañía con estilo de venta directa: mercadeo y supply chain. La creación de una aplicación automatizada garantiza la valoración, simulación y evaluación rápidas de diversos escenarios de ventas futuras, fundamentados en información histórica y cuantificación estadística de escenarios similares.

El departamento de estimados es el eslabón final de la cadena de mercadeo, puesto que al estimar se generan los reportes de forecast finales, los cuales deben estar en consonancia con las metas financieras planteadas originalmente por el departamento de marketing en la planificación. Así mismo, el área de estimados es el punto inicial de la cadena de suministros (o supply chain), en donde a través de las unidades estimadas, el área de compras procede a adquirir los insumos, componentes, ingredientes o productos terminados, a fin de garantizar el servicio final a la representante de ventas.

Debido a este alto nivel de responsabilidad, toda estimación por exceso o por defecto origina consecuencias realmente relevantes para ambas áreas: si se estima por exceso (Estimado final > Demanda real), se genera un exceso de inventario que afecta directamente la supply chain, y ocasiona un forecast estimado elevado, que no va en consonancia con la realidad de ingresos proveniente de la demanda real.

Ahora bien, si se estima por defecto (Estimado final < Demanda real), se genera un déficit en la cadena de suministros, la cual debe ser compensada por la "capacidad de reacción" que tenga la cadena, y el déficit será producto agotado para la representante, lo cual afecta a toda la organización tanto en términos de servicio como financieramente. En países con economías emergentes, la capacidad de reacción puede ser limitada debido a la situación macroeconómica, y así una gran cantidad de productos "subestimados" son inevitablemente agotados.

Se debe reconocer que la mayoría de las áreas que requieren modelos predictivos tienen en común la dinámica variante de sus actividades en el plano temporal. En la presente investigación esta última consideración se toma en cuenta en la estructura de ajuste de los residuos en el tiempo, en virtud de que la demanda tiene un patrón o tendencia asociada al tiempo, que puede cambiar a corto, mediano o largo plazo.

Finalmente, un sistema automatizado de proyección, no se debe concebir como un competidor del estimador o planificador de la demanda, sino más bien como una herramienta fundamental, que le permita a éste (usuario) realizar consultas en tiempo real, con la versatilidad 
de simular matemáticamente escenarios futuros, mediante modelos estadísticos que cuantifican la información histórica mediante de técnicas anteriormente expuestas, pero que nunca podrán valorar exactamente las creatividades de las nuevas estrategias empresariales de mercadeo y ventas.

\section{REFERENCIAS}

Allen, P. (1985). Ventas y dirección de ventas. España: EDAF, Ediciones Distrib.

Boada, A. (2000). Estimar la demanda de productos de línea regular en empresas con estilo de venta directa, bajo variables de mercadotecnia. Memorias del X Congreso Latino-Iberoamericano de Investigación de Operaciones y Sistemas. México.

Boada, A. (2002). Utilización del modelo lineal dinámico bayesiano de orden 1 como complemento de los residuos para modelos predictivos. Memorias del XI Congreso Latino-Iberoamericano de Investigación de Operaciones y Sistemas CLAIO'2002. Ciudad de Concepción, Chile.

Boada, A. (2011). Planificación de demanda, en empresas con estilo de venta por catálogo. Revista Lasallista, 8(2), 124-135. Indexada en el Publindex. Latindex. Scielo. Artículo invitado. ISSN 1794-4449

Boada, A. (2012). Diseño de un modelo de predicción de demanda, ajustado a empresas de estilo de venta directa. Memorias arbitradas en VIII Congreso de Investigación y Creación Intelectual de la Unimet. Caracas, Venezuela.

Boada, A. (2013). Modelo estadístico de regresión múltiple, columna vertebral para predecir en empresas multinacionales con estilo de venta por catálogo. Revista Lasallista, 10(1), 112-127. Indexada en el Publindex. Latindex. Scielo. Artículo invitado. ISSN 1794-4449.

Chan-Kim, W. (1990). La estrategia del océano azul. Bogotá: Norma.

Chase, R., Jacobs, R. \& Aquilano, N. (2005). Administración de la producción y operaciones para una ventaja competitiva (10… ed.). México: McGraw-Hill.

Dallas E., J. (1998). Applied Multivariate Methods for Data Analysis. Michigan: Brooks Cole Publishing Company.

Diebold, F. (1998). Elements of Forecasting. Mason: South Western College Publishing.

Lind, D. (2015). Estadística aplicada a los negocios y la economía (16a. ed.). México: McGraw-Hill.

Pericchi, L. R. (2002). Análisis de decisión, inferencia y predicción estadística bayesiana. Universidad Simón Bolívar.

Peter, J. P. (2006). Comportamiento del consumidor y estrategia de marketing (7ä. ed.). México: McGraw-Hill.

Prieto H., J. E. (2013). Investigación de mercados (2ª ed.). México: Ecoe.

Schiffman, L. \& Kanuk, L. L. (1991). Comportamiento del consumidor (3a a ed.). México: Prentice Hall.

Stanton, W. J. (2007). Fundamentos de Marketing (14ㄹ. ed.). México: McGraw-Hill.

Webster, A. L. (2000). Applied Statistics for Business and Economics ( $3^{\mathrm{a}}$. ed.). México:The McGraw-Hill Companies, Inc.

West, M., \& Harrison, J. (1989). Bayesian Forecasting and Dynamic Models. New York: Springer-Verlag. 
